\section{$\underset{\substack{\text { hommes } \\ \text { \& migrations }}}{ }$}

\section{Hommes \& migrations}

Revue française de référence sur les dynamiques

migratoires

\section{9 | 2009}

L'Afrique en mouvement

\title{
Migrants et insécurité existentielle
}

Le cas de Brazzaville

\section{Bruce Whitehouse}

\section{(2) OpenEdition}

1 Journals

\section{Édition électronique}

URL : http://journals.openedition.org/hommesmigrations/317

DOI : 10.4000/hommesmigrations.317

ISSN : 2262-3353

Éditeur

Musée national de l'histoire de l'immigration

\section{Édition imprimée}

Date de publication : 1 mai 2009

Pagination : 80-87

ISSN : 1142-852X

\section{Référence électronique}

Bruce Whitehouse, « Migrants et insécurité existentielle », Hommes \& migrations [En ligne], 1279| 2009,

mis en ligne le 29 mai 2013, consulté le 30 avril 2019. URL : http://journals.openedition.org/

hommesmigrations/317 ; DOI : 10.4000/hommesmigrations.317 


\section{Migrants et insécurité existentielle \\ Le cas de Brazzaville}

Par Bruce Whitehouse, professeur assistant d'anthropologie, Lehigh University, Bethlehem (États-Unis)

Un peu partout sur le continent africain, les immigrés originaires d'Afrique de l'Ouest sont victimes de violences et de discriminations. En Afrique du Sud, en Côte d'Ivoire ou en République du Congo, le ressentiment monte parmi les populations autochtones. Dans un contexte de crise économique, elles reprochent aux migrants d'accaparer les emplois. C'est le cas à Brazzaville, où il est difficile pour les Ouest-Africains de s'intégrer. Plongés dans une société qui les rejette, ces migrants vivent une insécurité aux multiples visages. 
2005, un dimanche à Brazzaville. Dans le marché de Poto-Poto, normalement bouillonnant d'activités, règne un calme inhabituel. Déjà à midi, les boutiques sont presque toutes fermées, les propriétaires ont bouclé leurs volets métalliques avant de regagner hâtivement leurs maisons. Des centaines de commerçants étrangers - maliens pour la plupart, ainsi que des Mauritaniens, Guinéens, Sénégalais, Béninois et Tchadiens - ont déserté le marché et, puisque ces étrangers africains dominent la vente des produits importés en ville, il n'y a que très peu d'entreprises ouvertes. On craint la violence. Pas, cette fois-ci, une reprise de la violence ethnorégionale qui a déchiré la République du Congo et dévasté sa capitale à plusieurs reprises dès le début de sa transition politique en 1992, puisqu'un accord de paix entre le gouvernement du président Dénis Sassou-Nguesso et un mouvement rebelle tient depuis 2003. Il s'agit ce dimanche d'une autre menace. Au stade Massamba-Débat, à quatre kilomètres du marché, un match de football oppose les Diables rouges, l'équipe nationale congolaise, aux Lions du Teranga, l'équipe nationale du Sénégal. Selon des rumeurs sinistres, les anciens "Cobras" (miliciens privés du Président) vont piller les maisons et les boutiques des immigrés ouest-africains si les Diables perdent. Inquiétés par la possibilité de représailles contre leur communauté, beaucoup d'Ouest-Africains n'osent pas sortir. Au stade, 3000 policiers et gendarmes se mobilisent afin d'empêcher la violence autour d'un évènement désigné par un journal local comme le "match de tous les dangers ${ }^{(1)}$ ".

Heureusement, à la suite d'un match nul, on évite le pire. Le lendemain, la vie reprend son cours à Brazzaville, sans émeutes ni pillages. Les inquiétudes des immigrés et des autorités étaient-elles justifiées? Quels dangers existent pour les migrants dans les pays africains? Ces questions exigent une étude des zones de conflit entre indigènes et immigrés. La ville de Brazzaville et sa population originaire de l'Afrique de l'Ouest sahélienne ont fait l'objet d'une étude de cas. Les membres de cette communauté vivent dans une insécurité permanente, croyant qu'il n'existe aucun remède contre leur exclusion de la société d'accueil. Nous verrons qu'il n'y a rien d'inhabituel dans ces perceptions. Du Caire au Cap, les relations entre immigrés africains et autochtones sont aujourd'hui de plus en plus tendues. Il n'y a rien d'unique dans les inhumanités xénophobes perpétrées en 2008 en Afrique du Sud ${ }^{(2)}$.

\section{Des immigrés soupçonnés}

Ce n'est pas un problème nouveau : l'histoire de l'Afrique postcoloniale regorge d'incidents de violence xénophobe ${ }^{(3)}$, dont plusieurs déclenchés par le football. En 1962, un match à Brazzaville entre les équipes nationales du Congo et du Gabon 
entraîne des violences contre les immigrés gabonais au Congo, suivie par des représailles contre les Congolais au Gabon. Dans les deux pays, neuf personnes sont tuées et plusieurs milliers d'immigrés expulsés. À Brazzaville, la "Rue des Gabonais" est par la suite officiellement rebaptisée la "Rue des Martyrs". En 1981, des bagarres lors d'un match à Libreville entre équipes professionnelles du Cameroun et du Gabon conduisent à l'oppression des immigrés camerounais, dont 10000 sont obligés de fuir le territoire gabonais ${ }^{(4)}$. En 1993, à Abidjan, des centaines d'immigrés ghanéens sont lynchés suite à la défaite de l'équipe nationale ivoirienne devant l'équipe du Ghana ${ }^{(5)}$. En 2005, en Zambie, des émeutes détruisent une mosquée ainsi que plusieurs voitures et boutiques après la défaite de l'équipe nationale zambienne face au Sénégal ; un Malien est gravement blessé( ${ }^{(6)}$.

Force est de constater que dans plusieurs pays africains, l'immigration est perçue comme un problème sociopolitique de plus en plus grave. Si le Congo a été épargné par la violence xénophobe massive, des tensions importantes existent entre Congolais et étrangers. La communauté ouest-africaine de Brazzaville est l'objet de l'hostilité générale, à cause notamment de son emprise sur le secteur commercial local. Cette emprise est aussi vieille que la ville ${ }^{(7)}$. Néanmoins, les dynamiques économiques et politiques contemporaines (à savoir, une crise économique aiguë, l'endettement et la contraction du secteur public, la corruption des organismes de l'État) font que les Congolais supportent de moins en moins les activités des entrepreneurs étrangers sur leur sol. Une enquête menée auprès des universitaires à Brazzaville montre que la grande majorité voit les Ouest-Africains au Congo comme malhonnêtes, corrupteurs, et nuisibles au développement économique nationa ${ }^{(8)}$. Pour beaucoup de Brazzavillois, les immigrés seraient responsables de la criminalité, de la violence et de l'insalubrité de leur ville.

\section{Floraison oppositionnelle}

Les immigrés ouest-africains se considèrent, pour leur part, victimes d'une discrimination systématique et de la corruption de la société d'accueil. Les policiers et gendarmes commettent des exactions de façon quotidienne ; les femmes sont fréquemment insultées dans la rue (leur mode vestimentaire les distingue comme musulmanes, donc étrangères). Les commerçants et entrepreneurs ouest-africains sont obligés de payer des redevances et taxes parafiscales en tout genre aux agents de l'État congolais. Et ils ne protestent pas, pensant que les étrangers n'en ont pas le droit. Même si les Congolais sont, eux aussi, victimes de cette gabegie, les OuestAfricains la vivent plus violemment, car elle fait écho dans leur histoire : ils sont 
toujours hantés par le souvenir de septembre 1977, quand ils subirent la nationalisation de leurs entreprises et l'expulsion manu militari vers leurs pays d'origine(9). De par leur histoire collective, les Ouest-Africains se considèrent mal accueillis au Congo. Ils y investissent peu, préférant remettre la grande partie de leurs bénéfices aux pays d'origine, où ils sont à l'abri de l'expropriation. "Le Congo ne veut pas intégrer les gens. Comment vous pouvez investir dans un pays là où vous n'êtes pas intégré ?' demande Madu, 40 ans, vendeur malien de pièces de rechange. "Chaque jour tu sors de chez toi, tu te sens étranger." Entre autochtones et étrangers les relations se durcissent, le cycle de discrimination et de méfiance s'aggrave.

Dans ce contexte politico-économique, les intérêts communs des deux populations, indigène et immigrée, sont progressivement masqués, alors que les différences entre leurs membres sont exacerbées. Si les transactions entre les opérateurs économiques étrangers et leurs clients congolais continuent, les frontières sociales et culturelles qui les séparent deviennent difficiles à franchir. Les avis des uns et des autres se caractérisent par des stéréotypes totalisants. Une dynamique de "floraison oppositionnelle ${ }^{(10) !}$ nécessite la sélection stratégique de symboles culturels mettant l'accent sur les particularités de chaque groupe. Même en l'absence de toute distinction raciale, l'écart perçu entre Congolais et Ouest-Africains à Brazzaville paraît de plus en plus inconciliable. 


\section{L'environnement structurel de l'hostilité}

Au début des années quatre-vingt, grâce à sa production de pétrole, le Congo était sur le point de rejoindre les pays à revenus moyens. Cependant, au cours des deux décennies passées, son climat politique et économique s'est dégradé suite à la diminution des recettes pétrolières. Durant cette période, le revenu par tête d'habitant a diminué de $30 \%{ }^{(11)}$. Dans les années quatre-vingt-dix, le pays a été saigné par la guerre civile et une transition démocratique échouée; même la hausse du cours mondial du pétrole depuis 2001 n'a pas pu arrêter son déclin. Face à l'endettement public massif, la mauvaise gestion de la richesse pétrolière, le chômage et la pauvreté généralisée, le pouvoir congolais s'est réjoui en 2006 de la classification du pays comme PPTE (pays pauvre très endetté) par la Banque mondiale ${ }^{(12)}$.

Aujourd'hui, la fonction publique, ancienne épine dorsale de l'emploi national, ne recrute plus, et le salaire d'un fonctionnaire est presque sans valeur. Dans les quartiers de Brazzaville, les coupures de courant et d'eau sont banales, la pénurie de carburant aussi. Le taux de scolarisation est en baisse depuis quinze ans, aussi bien en raison du manque de moyens des parents que du manque de travail pour les diplômés. Face au blocage de ses aspirations au développement, la population congolaise vit la déchirure totale.

Quel que soit le pays, de telles conditions matérielles risquent d'aiguiser les sentiments xénophobes. Les ressentiments populaires à Brazzaville contre les immigrés ouest-africains (surtout les commerçants) existent depuis longtemps, mais deviennent de plus en plus insupportables. Même s'il y a assez peu de grands opérateurs économiques parmi ces entrepreneurs étrangers, leur prospérité, comparée à l'appauvrissement des Congolais, suscite des soupçons et des expressions nationalistes. Beaucoup de Congolais estiment que les Ouest-africains mènent une existence parasitaire, et que leur succès est dû au favoritisme des autorités corrompues. Selon eux le Président et ses proches ont vendu le pays aux étrangers, en l'occurrence aux Ouest-Africains. Les Congolais se sentent exclus dans leur propre pays ${ }^{(13)}$. "Souvent, les Congolais pensent que les étrangers sont comme des voleurs de passage", dit Moussa, Guinéen âgé de 60 ans. "Ils ne comprennent pas que quand un Ouest-Africain vient ici, il fait la manutention, le colportage. Ce sont des boulots que les Congolais ne feraient pas."

\section{Légalité et illégalité toutes confondues}

Conséquence de l'écroulement de l'État ces dernières années, la question des formalités administratives est devenue problématique pour les immigrés à Brazzaville. Si les papiers qui légitiment le séjour d'un étranger africain sur le sol congolais sont toujours 
disponibles, ils ne présentent plus tellement d'avantages pour celui qui les porte. Il est devenu si facile d'obtenir des faux papiers (acte de naissance, carte d'identité, passeport) - voire des papiers authentiques par des moyens illégitimes - que le document officiel a perdu beaucoup de sa valeur. Les enfants d'immigrés, nés au Congo, donc détenteurs de la nationalité congolaise, ont souvent du mal à prouver leur statut de congolais à cause de leurs noms étrangers. "Quand [les policiers] t'arrêtent pour voir tes documents, ils disent 'Un Congolais qui s'appelle Sékou Mamadou, qui a vu ça?’' dit un jeune homme de 22 ans, né à Brazzaville de parents maliens. Ibrahim, commerçant malien de 50 ans (dont vingt-trois passés au Congo), raconte que son "frère cadet est né ici". "Il avait des papiers congolais, mais un jour, à la sortie de l'aéroport de Maya-Maya [à Brazzaville], on les a confisqués. On lui a dit que son passeport était faux... Depuis, il ne l'a pas récupéré." L'illégalité n'a aucun sens en l'absence d'un État de droit. Au Congo la distinction entre migration légale et migration clandestine est quasiment effacée. Pour l'immigré africain, sa carte de séjour n'empêche nullement les policiers et autres agents de l'État d'extorquer son argent ou de le mettre en détention. Pourquoi payer les frais de la carte (100 000 francs CFA par an, soit 150 euros) si on continue de subir des exactions régulières? Dans une société rongée par la corruption, "il n'y a pas accord préalable ni paiement une fois pour toutes, qui permettraient ensuite d'obtenir ce que l'on souhaite (telle ou telle pièce administrative, droit de circuler...), mais une sorte d'interminable négociation avec une multitude d'intervenants ${ }^{(14) "}$.

Il faut souligner qu'au Congo, comme dans d'autres pays de la région ${ }^{(15)}$, ce système informel extra-legal de contrôle territorial et de discrimination semble s'appliquer principalement aux étrangers africains; les expatriés blancs et asiatiques sont, de manière générale, épargnés. En effet, j’ai pu observer chaque jour lors de mes douze mois de recherches à Brazzaville que mes voisins et amis ouest-africains étaient "contrôlés" systématiquement, alors qu'on n'a exigé la pièce d'identité de l'Américain blanc que je suis une seule fois.

\section{Insécurité existentielle et boucs émissaires}

Les immigrés ouest-africains à Brazzaville vivent aujourd'hui dans ce que j'appelle un état d'insécurité existentielle. Pour eux, l'étranger africain ne peut jamais être à l'aise sur le sol congolais. Sa vie sera peut-être un jour interrompue de manière insolite et arbitraire simplement à cause de son statut d'étranger. Une relance de la guerre, un changement de gouvernement ou même un match de football... L'émigré risque à tout moment de perdre tous ses biens et de se voir expulsé du pays. "Pour un Congolais, tout immigré dans son pays peut être chassé n'importe quand. Il faut s'attendre au rapatriement à tout moment", affirme Tiékoro, immigré malien de 70 ans. "Ceux qui sont 
venus récemment ne comprennent pas, mais moi jai vécu les évènements de 1977, je comprends." Il n'y a aucun document et aucun processus de naturalisation qui puisse totalement dissoudre cette insécurité, qui se transmet d'ailleurs de génération en génération. Pour beaucoup d'enfants et petits-enfants d'immigrés africains, l'intégration à la société congolaise reste toujours partielle, sinon inaccessible. KadiFatou, 44 ans, fille d'un père sénégalais et d'une mère congolaise, se trouve bloquée dans sa carrière de fonctionnaire. "Quand tu es étranger [sic] travaillant dans une administration, il y a toujours une différence, tu vois?...Tu peux mériter une position à la direction, mais la première chose qu'ils verront, c'est que tu es étranger. Même avec la nationalité congolaise, avec ton nom... quelqu'un avec un nom congolais aura plus de chance que toi." L'influence croissante des appartenances ethniques et régionales sur la gestion de l'État fait que la nationalité et la citoyenneté moderne au Congo sont aujourd'hui presque dénuées de signification. De plus en plus, l'identité nationale se base non pas sur un fond bureaucratique (documents officiels, etc.) mais sur l'autochtonie. C'est le sang et pas le passeport qui fait le Congolais, surtout quand ce sang s'accompagne d'un patronyme du pays.

Les Ouest-Africains de Brazzaville savent bien que cette question dépasse les frontières congolaises. Confrontés à l'impossibilité de l'intégration, ils s'attendent à des mauvais traitements un peu partout sur le continent. "Tu sais, jai vécu au Togo, au Ghana, c'est toujours la même chose entre les immigrés et les indigènes", affirme Fanta, Malienne de 22 ans, déjà forte de dix années d'expérience de vie émigrée. "S'il y a une dispute entre toi et un Ghanéen [au Ghana] et tu te plains aux autorités, rien n'arrivera. C'est pareil au Togo et au Bénin".

Dans beaucoup de pays africains, l'immigré est perçu comme propagateur de fléaux aussi bien sociaux qu'économiques. C'est toujours l'étranger qui est accusé d'avoir introduit le sida : à Brazzaville, ceux qui ont été accusés étaient les ressortissants de la République démocratique du Congo (toujours appelés "Zaïrois" par les Brazzavillois) ${ }^{(16)}$. De même pour le vol : "Presque tous les vols commis à Brazzaville étaient imputés à des Zaïrois car leur pays symbolisait le règne de la délinquance et de la criminalité urbaines $^{(17)}$." En Afrique du Sud, les immigrés africains sont tenus responsables non seulement du vol des emplois mais également de la prostitution et du crime organisé $^{(18)}$. Ainsi, dans les sociétés d'accueil africaines, selon les mots d'un chercheur gabonais, "au mythe de la spoliation économique s'ajoute celui de la perversion sociale d'origine étrangère (19)". Même dans un pays d'émigration importante comme le Mali, où "l'aventure" et la vie migrante sont depuis longtemps des éléments intégrés au tissu social, les étrangers africains sont les premiers à être accusés d'activités sinistres de la plus absurde variété, une hystérie à l'égard des "rétrécisseurs de sexe" balaie ainsi périodiquement la région ${ }^{(20)}$. Le mal arrive toujours d'Ailleurs. On observe davantage en Afrique une "tendance croissante vers l'autochtonie comme 
critère décisif pour les droits sous l'État postcolonial'(21). Étant donné l'écroulement de la citoyenneté dans beaucoup de ces pays, on peut être citoyen dans tous les sens du terme - bénéficier de la sécurité durable, participer à la vie politique, devenir candidat - uniquement là où l'on réussit à démontrer une affiliation dite ancestrale. L'insécurité existentielle des immigrés africains aujourd'hui découle surtout de cette tendance à favoriser l'autochtonie. De là, un paradoxe fondamental pour un grand nombre de migrants sur le continent : si la vie chez soi est rendue impossible par la sécheresse, le conflit ou le manque d'emploi, c'est seulement "là-bas" que l'on peut vraiment se considérer... "chez soi".

\section{Notes}

1. "Congo-Sénégal, un match de tous les dangers?", La Semaine Africaine $\mathrm{n}^{\circ} 2521,2$ juin 2005.

2. Aurelia Wa Kabwe-Segatti, "Violences xénophobes en Afrique du Sud : retour sur un désastre annoncé", Politique africaine, $\mathrm{n}^{\mathrm{O}} 112,2008$.

3. Marc-Antoine Pérouse de Montclos, "Violences xénophobes en Afrique", Manière de voir, no 62, 2002.

4. Constant-Félix Pambo-Loueya, "Les étrangers et le travail au Gabon : rapatrier... unique solution ?" In Catherine Coquery-Vidrovitch et al., Être étranger et migrant en Afrique au $X X^{e}$ siècle : enjeux identitaires et modes dinsertion,

Vol. 2 : Dynamiques migratoires, modalités d'insertion urbaine et jeux d'acteurs, Paris, L'Harmattan, 2003.

5. Robert Calderisi, The Trouble with Africa: Why Foreign Aid isn't Working, New York, Palgrave MacMillan, 2006, p. 119.

6. BBC, 5 septembre 2005, "Zambian Fans go on the Rampage", http://news.bbc.co.uk/go/pr/fr/-/ sport2/hi/football/africa/4213240.stm.

7. Les anciens tirailleurs sénégalais étaient les premiers commerçants africains à s'installer dans la capitale coloniale ; voir Charles Gondola, Villes Miroirs : migrations et identités urbaines à Kinshasa et Brazzaville, 1930-1970, Paris,

L'Harmattan, 1996.

8. Bruce Whitehouse, "Exile Knows no Dignity: African Transnational Migration and the Anchoring of Identity," thèse doctorale, Brown University, 2007 et "Discrimination, Despoliation and Irreconcilable difference: HostImmigrant Tensions in Brazzaville, Congo", Africa Spectrum, vol. 2009, nº 1, 2009.

9. Le rapatriement forcé des immigrés sert depuis longtemps aux États africains à régler certains problèmes politiques internes ; voir Marc-Antoine Pérouse de Montclos, "L'Afrique rejette ses propres immigrés", Le Monde diplomatique, décembre 1995.

10. Voir Joshua Roth, Brokered Homeland: Japanese Brazilian Migrants in Japan, Ithaca, Cornell University Press, 2002, p. 140.

11. FMI, Country Report no. $05 / 391,2005$. http://www.imf.org.

12. Les Dépêches de Brazzaville, 9 mars 2006, "Fonds monétaire international : le Congo accède au 'point de décision' de l'Initiative pays pauvres très endettés". http://www.brazzaville-adiac.com.

13. Voir les discussions de l'immigration au Congo sur les sites Mwinda (www.mwinda.org) et Congopage (congopage.com/forums).

14. Patrick Chabal et Jean-Pascal Daloz, L'Afrique est Partie! Du désordre comme instrument politique, Paris, Economica, 1999 , p. 126.

15. Voir l'analogue en Afrique du Sud décrit par Loren Landau et Tamlyn Monson, "Displacement, Estrangement and Sovereignty: Reconfiguring State Power in Urban South Africa", Government and Opposition, vol. 43, no 2, 2008. 16. Didier Fassin, "Le domaine privé de la santé publique : pouvoir, politique et sida", Annales : Histoire, Sciences Sociales, vol. 49, no 4, 1994.

17. Rémy Bazenguissa-Ganga, "Instantanés au cceur de la violence : anthropologie de la victime au Congo-Brazzaville", Cahiers d'études africaines, vol. $38, \mathrm{n}^{\mathrm{O}} 2-4,1998$, p. 622.

18. Antoine Bouillon éd., Immigration africaine en Afrique du Sud : les migrants francophones des années 90, Paris, Karthala, 1999. .

19. Serge Loungou, "Immigration et Xénophobie au Gabon", Géopolitique Africaine 10, 2003, p. 258 ; voir également Pérouse de Montclos, 2002.

20. Jean-Jacques Mandel, "Les rétrécisseurs de sexe : chronique d'une rumeur sorcière", Cahiers d'Études Africaines, vol. $48, n^{\circ} 1-2,2008$.

21. Mahmood Mamdani, "Beyond Settler and Native as Political Identities: Overcoming the Political Legacy of Colonialism", Comparative Studies of Society and History, vol. 43, no 4, 2001, p. 657 ; voir également Armand Cutolo et Peter Geschiere éds., "Enjeux de l'autochtonie", Politique africaine, no 112, 2008. 